\title{
Endogenous retroviruses: from infectious elements to bona fide genes with a physiological role
} Thierry Heidmann*1,2, Marie Dewannieux ${ }^{1,2}$, Anne Dupressoir ${ }^{1,2}$, Cécile Esnault ${ }^{1,2}$, Odile Heidmann ${ }^{1,2}$, Christian Lavialle ${ }^{1,2}$, Géraldine Louf ${ }^{1,2}$, David Ribet ${ }^{1,2}$ and Cécile Vernochet ${ }^{1,2}$

Address: ${ }^{1}$ CNRS UMR 8122, Institut Gustave Roussy, Villejuif, F-94805, France and ${ }^{2}$ Université Paris-Sud, Orsay, F-91405, France

* Corresponding author

from Frontiers of Retrovirology: Complex retroviruses, retroelements and their hosts

Montpellier, France. 2I-23 September 2009

Published: 24 September 2009

Retrovirology 2009, 6(Suppl 2):12 doi:10.1186/1742-4690-6-S2-12

This abstract is available from: http://www.retrovirology.com/content/6/S2/12

(C) 2009 Heidmann et al; licensee BioMed Central Ltd.

Endogenous Retroviruses (ERVs) are present in all higher eukaryotes where they represent a large fraction of the genomes ( $8 \%$ in humans, divided into about 100 families). They are remnants of ancestral infections by bona fide retroviruses that have infected the germline and have thereafter been transmitted in a Mendelian manner. Conversely, they are a reservoir from which new infectious retroviruses can arise via recombination. The similarity between ERVs and present-day infectious retroviruses is remarkable, in terms of genome organization, encoded proteins, structures of the associated virus-like particles, and to some extent replicative cycles. Although most ERVs are defective due to genetic drift, the "molecular biology" of functional elements that could be identified in the human and murine genomes will be presented. Some ERVs have retained infectious properties (e.g. the HERV-K, GLN and IAPE elements), whereas others have evolved toward retroelements with a strictly intracellular life cycle (e.g. MusD and IAP elements) with high insertional mutagenesis activity. The molecular basis for these evolutionary transformations has been characterized, as well as the putative and/or still-present progenitors of these "intracellularized" elements. A step further to retrovirus "capture" by the host genomes will be exemplified with the socalled syncytin genes, which are retroviral envelope genes that have been ancestrally "hijacked" by several mammalian species - including primates and rodents- and have been conserved over $>30$ millions years of evolution in a functional state: syncytins have a placenta-specific expression and a cell-cell fusogenic activity most probably involved in placentation and syncytiotrophoblast formation. Data based on knockout mice will be presented that lend support to this subverted and essential role of ERV genes in mammalian physiology, and on the convergent impact of these independently acquired elements. Finally, the refined mechanisms that have evolved for the host control of ERVs and related elements will be described, with special emphasis on the restriction effects of the cellular APOBEC3 cytosine deaminase proteins, which inhibit amplification of both the infectious ERVs and the intracellularized retroelements, and whose effects can be clearly detected through the signatures imprinted on the numerous genomic copies of these elements.

\section{References}

I. Ribet D, Harper F, Dupressoir A, Dewannieux M, Pierron G, Heidmann T: An infectious progenitor for the murine IAP retrotransposon: emergence of an intracellular genetic parasite from an ancient retrovirus. Genome Res 2008, I 8:597-609.

2. Esnault C, Priet S, Ribet D, Heidmann O, Heidmann T: Restriction by APOBEC3 proteins of endogenous retroviruses with an extracellular life cycle: ex vivo effects and in vivo "traces" on the murine IAPE and human HERV-K elements. Retrovirology 2008, 5:75.

3. Esnault C, Priet S, Ribet D, Vernochet C, Bruls T, Lavialle C, Weissenbach J, Heidmann T: A placenta-specific receptor for the fusogenic, endogenous retrovirus-derived, human syncytin2. Proc Natl Acad Sci USA 2008, 1 05:17532-7.

4. Dupressoir A, Vernochet C, Bawa O, Harper F, Pierron G, Opolon $P$, Heidmann T: Syncytin-A knockout mice demonstrate the critical role in placentation of a fusogenic, endogenous retrovirus-derived, envelope gene. Proc Natl Acad Sci USA 2009 in press. 